\title{
Impact of the Keto-Enol Tautomeric Equilibrium on the BODIPY chromophore
}

Volker Leen, ${ }^{\mathrm{a}}$ Marina Laine, ${ }^{\mathrm{b}}$ Joseph Molisho Ngongo, ${ }^{\mathrm{c}}$ Paweł Lipkowski, ${ }^{\mathrm{d}}$ Bram Verbelen, ${ }^{\mathrm{a}}$ Andrzej Kochel, ${ }^{\mathrm{b}}$ Noël Boens, ${ }^{\mathrm{a}}$ Wim Dehaen, ${ }^{\mathrm{a}}$ Mark Van der Auweraer, ${ }^{\text {a }}$ Viktor Nadtochenko ${ }^{\mathrm{e}}$ and Aleksander Filarowski*b

${ }^{a}$ Department of Chemistry, KU Leuven, Celestijnenlaan 200f - bus 02404, 3001 Leuven, Belgium

${ }^{\mathrm{b}}$ Faculty of Chemistry, University of Wroclaw, F. Joliot-Curie 14, 50-383, Wrocław, Poland.

${ }^{c}$ Faculty of Science, Université de Kinshasa, B.P. 190, Kinshasa XI, Democratic Republic of the Congo

d Theoretical Chemistry Group, Institute of Physical and Theoretical Chemistry, Wrocław University of Technology, Wyb. Wyspiańskiego 27, 50-370 Wrocław, Poland

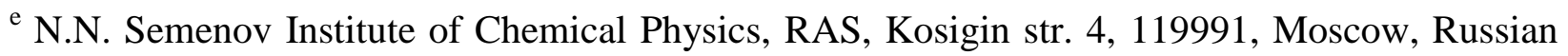
Federation

${ }^{\mathrm{f}}$ Department of Physics, Industrial University of Tyumen, 625-000, Tyumen, Russian Federation AUTHOR INFORMATION

\section{Corresponding Author}

*E-mail: aleksander.filarowski@chem.uni.wroc.pl 
ABSTRACT: An intramolecular tautomeric fluorescent BODIPY sensor has been designed and synthesized. The obtained BODIPY dye is a combination of the 4-bora-3a,4a-diaza $-s$-indacene core and a diketone fragment. The study of conformational equilibria in the ground and excited states has been completed for a broad range of solvent polarity by steady state and NMR methods as well as by DFT and TD-DFT calculations. The interpretation of the unique emission observed in hydrogen bond accepting solvents under the excitation of the fluorescent dye in the $\mathrm{S}_{0}-\mathrm{S}_{2}$ transition has been accomplished. The Jablonski diagram has been analyzed for the observed processes in the BODIPY dye studied on the basis of DFT and TD-DFT calculations.

\section{TOC GRAPHICS}

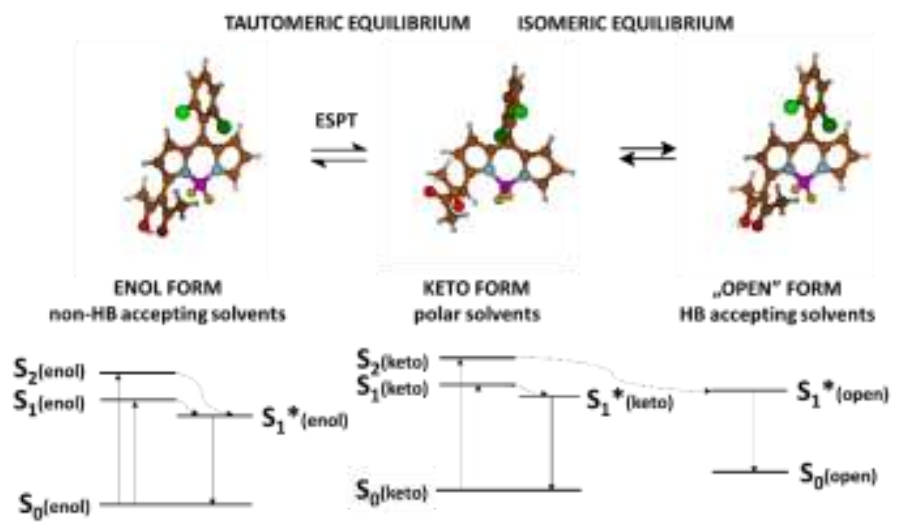

KEYWORDS BODIPY, diketone, keto-enol equilibrium, TD-DFT, conformational analysis, hydrogen bond.

TEXT. In this study we aim to report on the influence of a tautomeric sensor on the spectral characteristics of the difluoroborondipyrromethene (BODIPY) core in the ground and excited 
states based on the experimental results as well as quantum-mechanical calculations. The synthesis and spectroscopic characterization of fluorescent BODIPY dyes have been investigated extensively. ${ }^{1-3}$ These compounds are used for a large number of applications such as chromogenic probes and ions, ${ }^{4}$ fluorescent switchers, ${ }^{5}$ laser dyes, ${ }^{6} \mathrm{pH}$ indicators, ${ }^{7}$ and solar cells. $^{8}$ They are also employed in biochemical and biological applications. ${ }^{9,10}$ Several of these applications are based on the possibility to use fluorescence spectroscopy to measure intracellular concentrations of biologically important ions and molecules. Currently, the design, synthesis, and spectroscopic characterization of novel fluorescent probes continue to be important topics in this dynamic research area. ${ }^{11}$

In spite of extensive research on these dyes, only limited information is available on the impact of the tautomeric equilibrium and of hydrogen bonding on the charge transfer and spectral characteristics. ${ }^{12-15}$ Recently Akkaya et al. ${ }^{16,17}$ have shown the influence of protonation and deprotonation processes on the spectral characteristics of BODIPY dyes. Literature reports $^{13,15}$ investigated the influence of tautomeric equilibrium in ortho-hydroxy aryl Schiff bases substituted at the meso- or 7-position of the BODIPY core on the position of the absorption and emission bands as well as its influence on the fluorescent quantum yield. The later report ${ }^{15}$ shows that the replacement of the salicylideneaniline substituent with a naphthylideneaniline in the meso-position of the BODIPY dye brings about a visible decrease of the fluorescent quantum yield of the BODIPY-meso-Schiff dye (in methanol from 0.38 to 0.05 and in acetonitrile from 0.48 to 0.05$)$. The authors ${ }^{15}$ explained this phenomenon by the presence of a tautomeric equilibrium in the naphthalene derivative of the BODIPY-meso-Schiff dye, which results in a charge transfer (PET) from the Schiff fragment (in the ketoimine form) to the BODIPY core. The presence of a tautomeric equilibrium in the ground state is substantiated by changes of the 
absorption bands within the $300-450 \mathrm{~nm}$ region. ${ }^{15}$ It is necessary to note that the $\mathrm{S}_{0}-\mathrm{S}_{1}$ band stays similar upon transfer from a non-polar solute to a polar one (hexane to methanol). A different situation for the BODIPY-Schiff dye was observed as a result of substitution of the ortho-hydroxyaryl Schiff fragment at position 7 of the core. ${ }^{13}$ The change from a non-polar solute to apolar one triggers a hypsochromic shift of the main $\mathrm{S}_{0}-\mathrm{S}_{1}$ absorption band.

A recent paper by Lakshmi and Ravikanth ${ }^{18}$ analyzed the effect of phenylhydrazone and 2,4-dinitro-phenylhydrazone substituents on the spectral characteristics of BODIPY dyes. The paper revealed that the formation of an intramolecular hydrogen bond between the $-\mathrm{NH}-\mathrm{N}=\mathrm{CH}-$ $\mathrm{C}_{6} \mathrm{H}_{5}$ substituent and a hydrogen bonding accepting solvent (DMSO or DMF) causes a bathochromic shift of the absorption band $(50 \mathrm{~nm})$ and a significant shift of the ${ }^{1} \mathrm{H}$ NMR signal (about $3 \mathrm{ppm}$ ) towards low field. A stronger bathochromic shift of the absorption band (172 nm) was observed for the 2,4-dinitro-phenylhydrazone derivative of BODIPY as a result of the intramolecular $\leftrightarrows$ intermolecular hydrogen bond equilibrium, but, with a much smaller ${ }^{1} \mathrm{H}$ signal shift $(\sim 1 \mathrm{ppm})$. These phenomena were explained by the authors by an increase of the electron density on the nitrogen atom and a more stable delocalized structure.

To summarize, the influence of the intramolecular tautomeric equilibrium on the spectral characteristics of the BODIPY chromophore is an interesting topic for further investigations. Thus, the goal of this study is to have a deep understanding of the influence of the tautomeric equilibrium on the spectral characteristics of BODIPY dyes in the ground and excited states. The combination of the BODIPY core and a diketone substituent makes it possible to model the effect of the coupling between the quasi-aromatic $\mathrm{O}=\mathrm{C}-\mathrm{C}=\mathrm{C}-\mathrm{O}$ moiety and the chromophore core on the spectroscopic characteristics by changing the intramolecular hydrogen bonding (Scheme 1). 


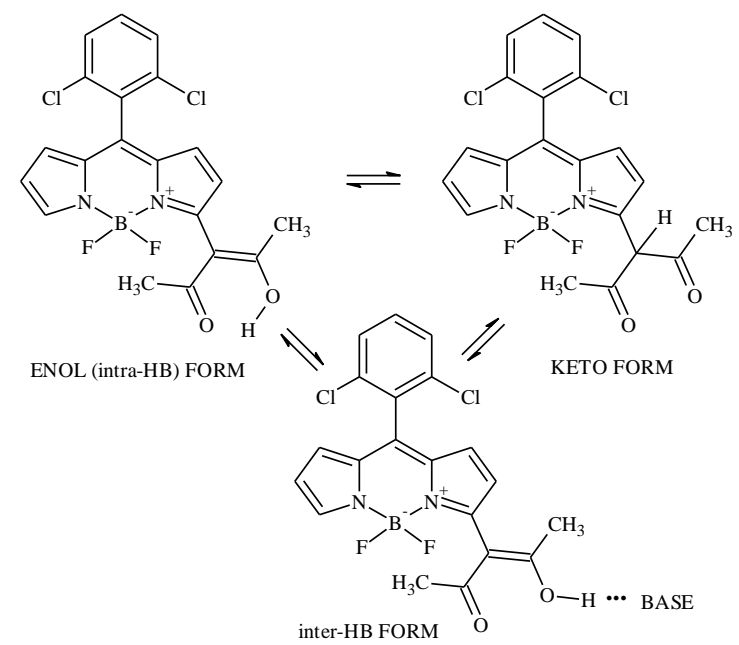

Scheme 1. Chemical structure of studied BODIPY dye and scheme of the tautomeric equilibria.

This investigation is expected to provide fundamental data for the effective modelling of the physicochemical properties of the chromophores. It is necessary to underline that the influence of hydrogen bonding on the fluorescent quantum yield is not unequivocal; on the one hand, hydrogen bonding diminishes the fluorescence quantum yield, but on the other hand, hydrogen bonding influences the charge transfer between the tautomeric sensor and the fluorophore, which can result in an increase of the fluorescence quantum yield. The elucidation of the role of the $\pi$ electronic conjugation between the chromophore and the quasi-aromatic substituent is a significant step forward and this phenomenon is studied here by the determination of the influence of solvent polarity on the photophysical properties of the diketone BODIPY dye.

The absorption spectra of the studied compound were characterized and showed a narrow absorption band with a maximum between $515 \mathrm{~nm}$ (trifluoroethanol) and $524 \mathrm{~nm}$ (toluene), with the exception of the hydrogen accepting solvents - acetone, acetonitrile, dimethylformamide and dimethylsulfoxide (Figure 1A). The absorption band has a shoulder at around $490 \mathrm{~nm}$ (Figure 1A), which can be attributed to vibrational progression. ${ }^{3}$ In the same series of solvents the emission maxima consist of a relatively narrow band without a fine structure and a maximum 
ranging from $533 \mathrm{~nm}$ (trifluoroethanol) to $545 \mathrm{~nm}$ (toluene). For the same range of solvents the Stokes shift amounts to $700 \pm 50 \mathrm{~cm}^{-1}$ and is nearly independent from solvent polarity. The spectral data as well as the fluorescence quantum yields (Table S1) suggest that in the solvents without hydrogen bonding character only a single absorbing and emitting species is present (prevailing enol tautomeric form, see discussion below, Scheme 1). However, in outspoken intermolecular hydrogen bond accepting solvents, acetone, acetonitrile, dimethylformamide and dimethylsulfoxide, the absorption spectrum consists of a broad unstructured band with maxima at respectively 539 and $542 \mathrm{~nm}$. Hence these maxima are red shifted compared to the nonhydrogen bond accepting solvents. This result shows that these hydrogen bond accepting solvents are characterized by two conformational forms of the molecule in the ground state. Excitation at the maximum of the $\mathrm{S}_{0}-\mathrm{S}_{1}$ band $(500-520 \mathrm{~nm})$ results in an intensive emission band within 540 - $550 \mathrm{~nm}$ (Figure 1). In acetone, acetonitrile, dimethylformamide and dimethylsulfoxide the features and maximum of the emission spectra still resemble those of the emitting species in nonhydrogen bond accepting solvents.

A

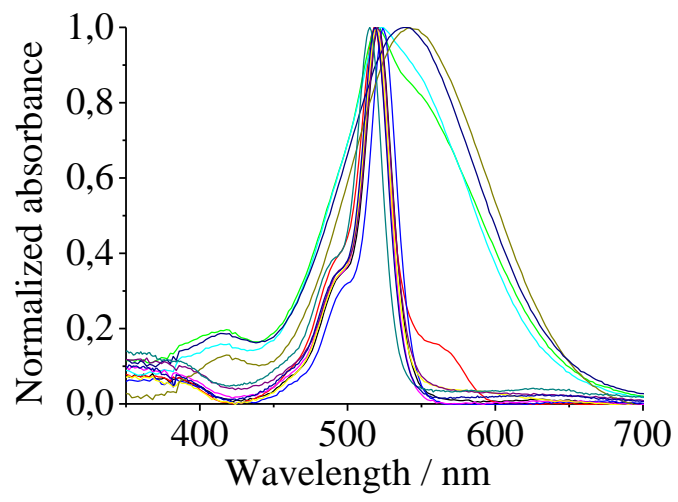

B

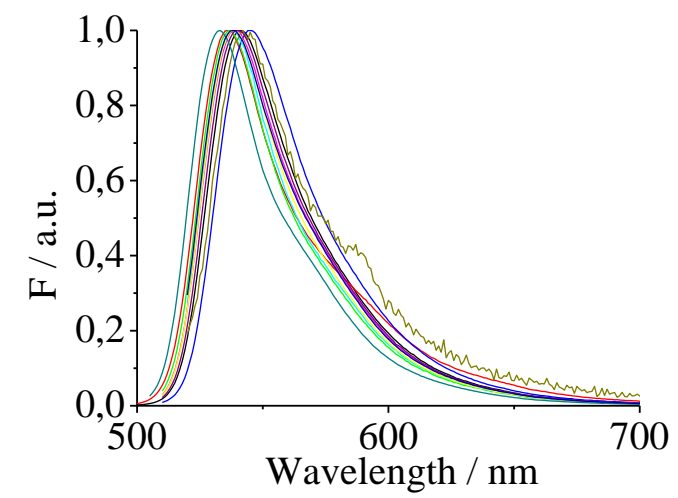

Figure 1. The normalized fluorescence (A) excitation spectra and (B) emission spectra of the studied BODIPY dye as function of solvent polarity: THF (black), methanol (red), $\mathrm{CH}_{3} \mathrm{CN}$ 
(green), toluene (blue), acetone (cyan), ethyl acetate (magenta), 2-propanol (yellow), DMSO (dark yellow), DMF (navy), 1-butanol (purple), TFE (dark cyan), acetic acid (royal).

These changes in absorption and emission accurately mirror the dependences $\lambda_{\mathrm{abs}} / \lambda_{\mathrm{em}}=\mathrm{f}(\Delta \mathrm{f})$ and $\Delta \bar{v}=\mathrm{f}(\Delta \mathrm{f})$ presented in Figure $2\left(\lambda_{\mathrm{abs}} / \lambda_{\mathrm{em}}=\mathrm{f}\left(\Delta \mathrm{E}_{\mathrm{N}}{ }^{\mathrm{T}}(30)\right)\right.$ and $\Delta \bar{v}=\mathrm{f}\left(\Delta \mathrm{E}_{\mathrm{N}}^{\mathrm{T}}(30)\right)$, Figure S2, supplementary). The linear dependencies presented in Figure 2 reveal that the increase of the solvent polarity (except for the solutions with hydrogen bond accepting solvents) evokes a quite insignificant hypsochromic shift of the absorption and emission bands. The results presented above prove that the studied compound does not undergo significant structural changes in these solvents (protic in that number). The hydrogen bond accepting solvents are the exception due to the increase of the shift of $\lambda_{\text {abs }}$ observed for these solvents.

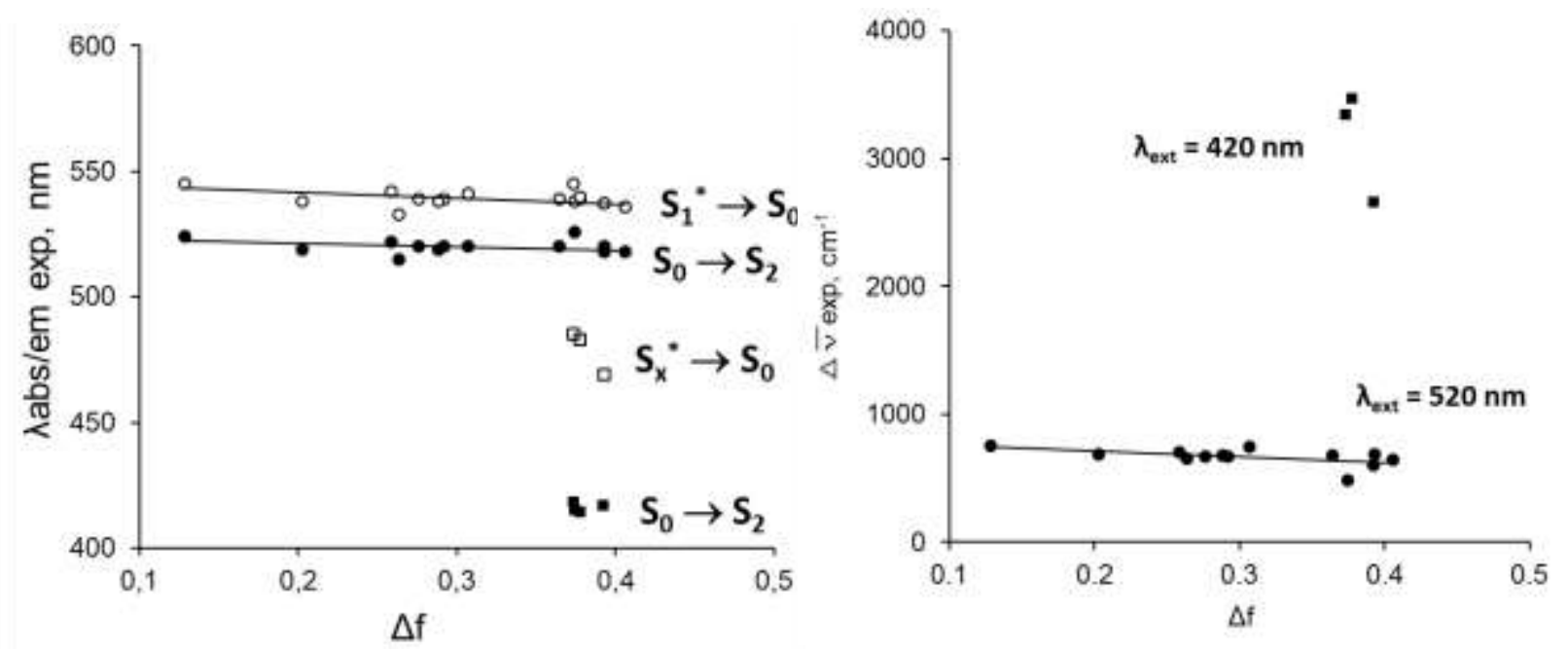

Figure 2. A) Black circles $\left(\lambda_{\text {abs }}\right)$ are in respect to the $S_{0} \rightarrow S_{1}$ transition (trend line $-\lambda_{\text {abs }}=-$ 14.299 $\Delta f+524.3)$; empty squares $\left(\lambda_{\text {abs }}\right)$ are in respect to $S_{0} \rightarrow S_{2}$ transition; empty circles $\left(\lambda_{\text {em }}\right)$ are in respect to $\mathrm{S}_{1} * \rightarrow \mathrm{S}_{0}$ (trend line $\left.\lambda_{\mathrm{em}}=-23.258 \Delta \mathrm{f}+546\right)$; empty squares $\left(\lambda_{\mathrm{em}}\right)$ are in respect to $S_{x}{ }^{*} \rightarrow S_{0}$. B) Black circles are in respect to the Stokes shift after excitation at $520 \mathrm{~nm}$ (trend 
line $\Delta \bar{v}=-462.01 \Delta f+806.67)$ and black squares are in respect to the Stokes shift after excitation at $420 \mathrm{~nm}$.

Therefore, the question arises: which tautomeric forms of the studied dye are observed in nonhydrogen bond accepting and an which in hydrogen bond accepting solvents in both the ground and the excited states? To answer this question ${ }^{1} \mathrm{H}$ NMR spectra have been measured in order to further identify these species in the ground state (Figure 3). All the NMR spectra recorded in toluene, chloroform and dichloromethane show an intense signal at $16.76 \mathrm{ppm}$ (chloroform), $16.69 \mathrm{ppm}$ (dichloromethane) and $17.44 \mathrm{ppm}$ (toluene). This signal is characteristic for an intramolecular hydrogen bond in the enol form of a $\beta$-diketone. ${ }^{19,20}$ This suggests that in nonhydrogen bond accepting solvents the dye is present only as the enol tautomeric form (intra-HB). Moreover, the absence of a band within the range of $4-5 \mathrm{ppm}$, diagnostic for the keto form of diketones, ${ }^{19,20}$ proves the lack of the keto form of the studied dye in non-hydrogen bond accepting solvents. However, hydrogen bond accepting solvents feature both the band within the range of $4-5 \mathrm{ppm}$ (THF - $4.62 \mathrm{ppm}$, acetonitrile $-5.40 \mathrm{ppm}$ and acetone $-5.49 \mathrm{ppm}$ ) and a significant decrease of the intensity of the band at $16-17 \mathrm{ppm}$. Notably, the ${ }^{1} \mathrm{H}$ NMR spectra of all the solutions do not reveal an additional wide band, which could point out the presence of an intermolecular hydrogen bond between the hydroxyl group of the dye and solvent molecules. The above- mentioned data make it clear that non-hydrogen bond solvents in the ground state exhibit only keto-enol equilibrium in the absence of a stable intermolecular hydrogen bond between BODIPY and the solvent molecules. 


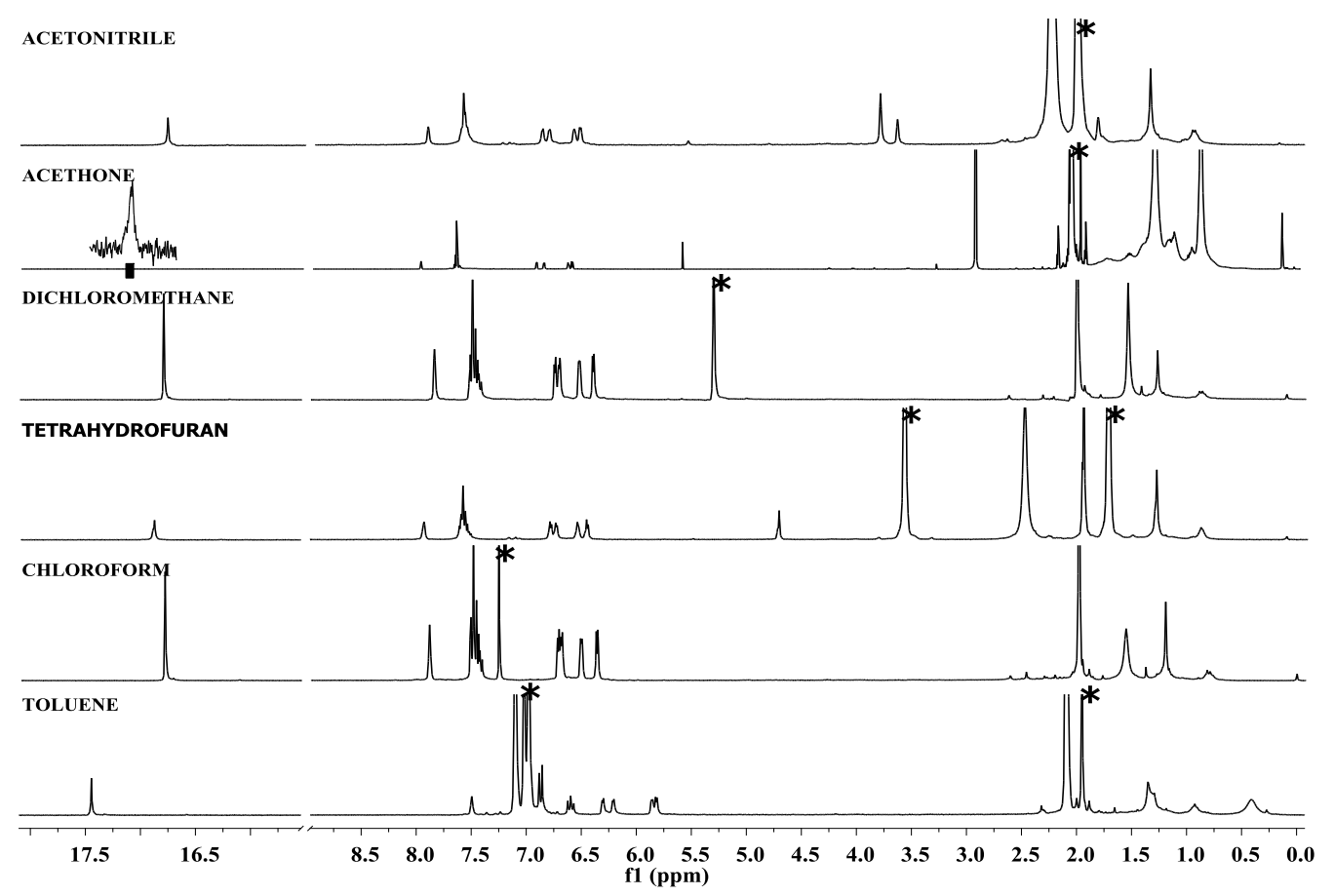

Figure 3. NMR spectra of 3-(2,4-dioxopentan-3-yl)-8-(2,6-dichlorophen-1-yl)-4,4-difluoro-4bora-3a,4a-diaza-s-indacene in acetonitrile (A), dichloromethane (B), tetrahydrofuran (C), chloroform (D) and toluene (E).

For the verification of the observed equilibria quantum-mechanical calculations have been accomplished for the dye in the ground and excited states. The computational analysis was carried out by $\mathrm{DFT}^{21}$ and TD-DFT ${ }^{22}$ methods for the ground and excited states, correspondingly. To make sure that the results are highly reliable, the calculations have been performed by four M06-2X, ${ }^{23}$ PBE0 $^{24}{ }^{24}$ CAMB3LYP ${ }^{25}$ and B3LYP ${ }^{26}$ functionals. All the calculations showed an analogous trend of the changes after the transition from the enol form to the keto form, except for the calculations by B3LYP functional for the gas phase which are not further discussed. The calculations provide 8 conformers for the ground and excited states (Figure 4). Besides, the calculations show that the conformer with an intramolecular hydrogen bond (conformer $\mathrm{C}$, 
Figure 4) is the least stable. The second most stable conformer is conformer C2 (the molecule in the keto form). The difference of energy between conformers $\mathrm{C} 1$ and $\mathrm{C} 2$ equals about $4 \mathrm{kcal} / \mathrm{mol}$ in the ground and excited states, respectively. The rest of the conformers, $\mathrm{C} 3-\mathrm{C} 8$, possess very similar energy in the ground and excited states $(10-15 \mathrm{kcal} / \mathrm{mol})$. As to the calculated spectral characteristics, the transition from the enol form (conformer C1) to the keto form (conformer C2) brings about a bathochromic shift of the absorption band and a hypsochromic shift of the emission band (Tables S3 - S5, supplementary). The results of the calculations are in good agreement with the observed experimental data. The calculations state that the difference of energy between conformer forms rapidly reduces with increasing solvent polarity (gas $\rightarrow$ dichloromethane $\rightarrow$ methanol $\rightarrow$ DMSO) especially for the exited state.
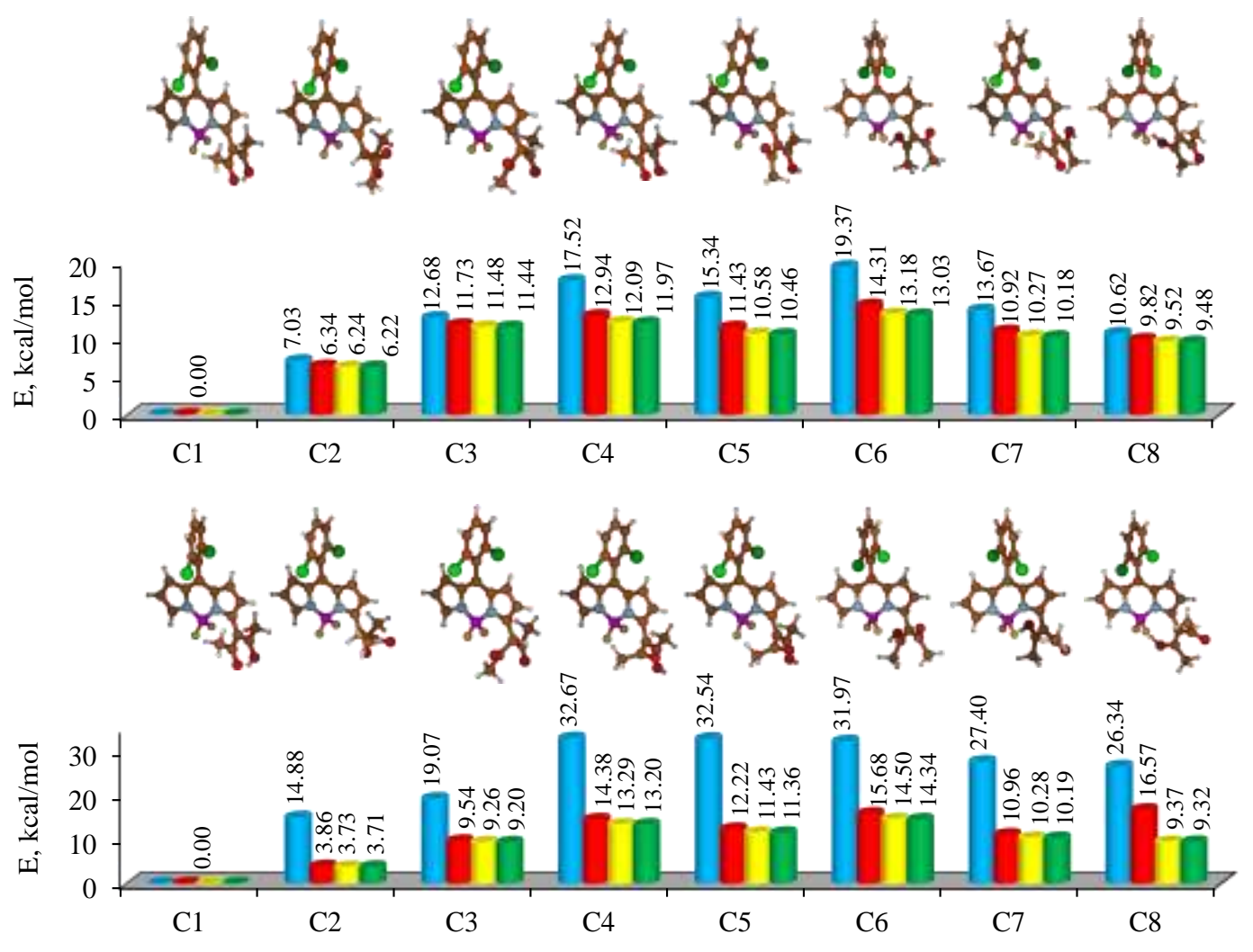
Figure 4. The energy and structure of conformers of obtained with (A) B3LYP/6-31+G(d,p) and (B) TD-B3LYP/6-31+G(d,p) for full optimisation parameters of molecule at ground and excited states, respectively. Gas (blue), dichloromethane (red), methanol (yellow) and DMSO (green).

The most interesting phenomenon for the studied compound seems to be the appearance of an absorption band within $400-450 \mathrm{~nm}$ in the hydrogen bond accepting solvents According to literature data, ${ }^{3}$ this band is assigned to a $\mathrm{S}_{0}-\mathrm{S}_{2}$ transition. It is noteworthy that the emission from $\mathrm{S}_{2}$ state has been observed before for BODIPY dyes. ${ }^{27-29}$ It is a surprising fact that the excitation at the $\lambda_{\max }\left(\mathrm{S}_{0}-\mathrm{S}_{2}\right)$ band of the studied dye causes a significant emission within the range of $469-486 \mathrm{~nm}$ with a large Stokes shift $\left(2500-3500 \mathrm{~cm}^{-1}\right.$, Table S1). The intensity of this emission band even exceeds the intensity of the band of $\mathrm{S}^{*}{ }_{1}-\mathrm{S}_{0}$ transition $(\sim 540 \mathrm{~nm}$, Figure 5). However, the obtained results differ from those reported earlier ${ }^{27-29}$ thanks to a significant intensity of the emission and a large (in terms of BODIPY dyes) Stokes shift. The explanation of this phenomenon is presented in Scheme 3 and analysed below.

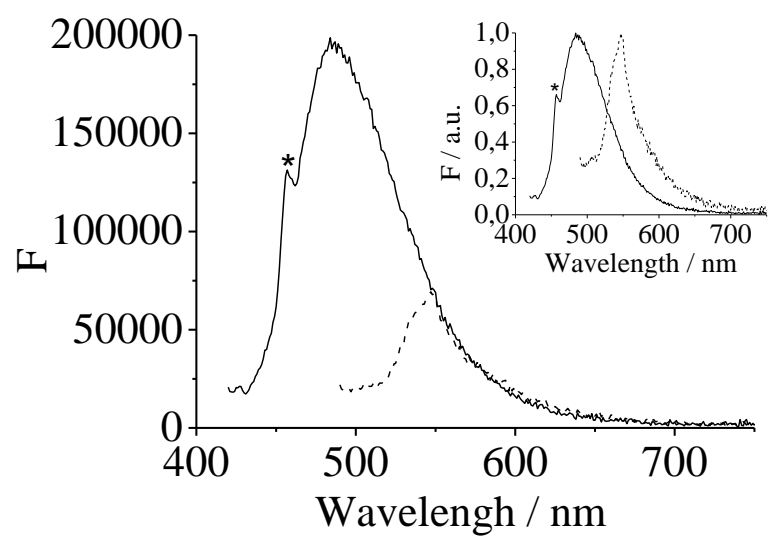

Figure 5. The fluorescence emission spectra; $\lambda_{\text {ext. }}=420 \mathrm{~nm}, \lambda_{\text {ext. }}=480 \mathrm{~nm}$ (inset normalized scale). 
This significant Stokes shift suggests important structural changes which are caused by the change of tautomeric form ${ }^{30,31}$ as a result of the transition from a $\mathrm{S}_{2}$ (keto form) state to an "unknown" $\mathrm{S} *_{1}\left(\mathrm{X}\right.$ ?) one. The "unknown" state $\mathrm{S} *_{1}\left(\mathrm{X}\right.$ ?) cannot be the $\mathrm{S}{ }_{1}($ keto) state or the $\mathrm{S} *_{1}(\mathrm{enol})$ state, otherwise one would observe the emission at $\lambda_{\mathrm{em}}=540 \mathrm{~nm}$, like in non-hydrogen bond accepting solvents. Therefore, the "unknown" $\mathrm{S}^{*}{ }_{1}(\mathrm{X}$ ?) state appears to be the excited state of the dye in the tautomeric "open" form, $\mathrm{S}^{*}{ }_{1}$ (“open"). It is important to mention that this tautomeric state of the dye is stabilized by the intermolecular hydrogen bonding between the hydroxyl group of the BODIPY dye and molecules of the hydrogen bond accepting solvent. Furthermore, the molecule undergoes a transformation from the $\mathrm{S}^{*}{ }_{1}(\mathrm{open})$ state to the ground state of the dye molecule with the open form $\left(\mathrm{S}_{0}(\right.$ open $\left.)\right)$ with a consequent non-radioactive transition into the $\mathrm{S}_{0}($ keto) state.

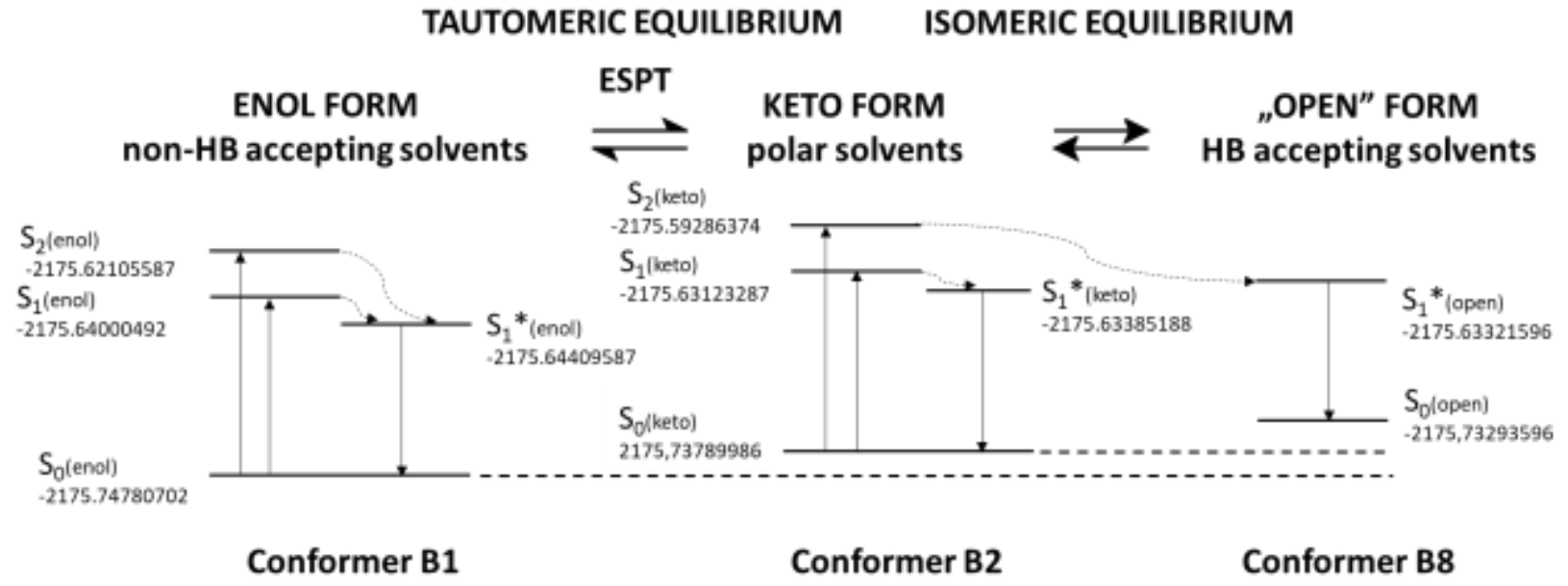

Scheme 3. Jabloński diagram of studied BODIPY dye.

\section{ASSOCIATED CONTENT}

(Word Style “TE_Supporting_Information”). Supporting Information. A listing of the contents of each file supplied as Supporting Information should be included. For instructions on what 
should be included in the Supporting Information as well as how to prepare this material for publications, refer to the journal's Instructions for Authors.

The following files are available free of charge.

brief description (file type, i.e., PDF)

brief description (file type, i.e., PDF)

\section{AUTHOR INFORMATION}

\section{Notes}

The authors declare no competing financial interests.

\section{ACKNOWLEDGMENT}

This work was supported by RFBR (no: 16-03-00405) and KNOW-12 grants. The authors gratefully acknowledge the Wroclaw Centre for Networking and Supercomputing (WCSS) for computational facilities.

\section{REFERENCES}

(1) Loudet, A.; Burgess. K. BODIPY Dyes and Their Derivatives: Syntheses and Spectroscopic Properties. Chem. Rev. 2007, 107, 4891-4932.

(2) Ulrich, G.; Ziessel, R.; Harriman, A. The chemistry of fluorescent bodipy dyes: versatility unsurpassed. Angew. Chem., Int. Ed. 2008, 47, 1184-1201.

(3) a) Boens, N.; Leen, V.; Dehaen, W. Fluorescent indicators based on BODIPY. Chem. Soc. Rev. 2012, 41, 1130-1172; b) Boens, N.; Verbelen, B.; Dehaen, W. Postfunctionalization of the BODIPY Core: Synthesis and Spectroscopy. Eur. J. Org. Chem. 2015, 6577-6595.

(4) Ueno, T.; Urano, Y.; Kojima, H.; Nagano, T. Mechanism-based molecular design of highly selective fluorescence probes for nitrative stress. J. Am. Chem. Soc. 2006, 128, 10640-10641. 
(5) Golovkova, T. A.; Kozlov, D. V.; Neckers, D. C. Synthesis and Properties of Novel Fluorescent Switches. J. Org. Chem. 2005, 70, 5545-5549.

(6) Chen, T.; Boyer, J. H.; Trudell, M. L. Synthesis of 2,6-diethyl-3-methacroyloxymethyl1,5,7,8-tetramethylpyrromethene-BF2 for the preparation of new solid-state laser dyes. Heteroat. Chem. 1997, 8, 51-54.

(7) Boens, N.; Qin, W.; Baruah, M.; De Borggraeve, W. M.; Filarowski, A.; Smisdom, N.; Ameloot, M.; Crovetto, L.; Talavera, E. M.; Alvarez-Pez, J. M. Rational design, synthesis and spectroscopic and photophysical properties of a visible-light-excitable, ratiometric, fluorescent near-neutral pH indicator based on BODIPY. Chem. Eur. J. 2011, 17, 10924-10934.

(8) Lammi, R. K.; Amboise, A.; Balasubramanian, T.; Wagner, R. W.; Bocian, D. F.; Holten, D.; Lindsey, J. S. Structural control of photoinduced energy transfer between adjacent and distant sites in multiporphyrin arrays. J. Am. Chem. Soc. 2000, 122, 7579-7591.

(9) Kowada, T.; Maeda, H.; Kikuchi, K. BODIPY-based probes for the fluorescence imaging of biomolecules in living cells. Chem. Soc. Rev. 2015, 44, 4953-4972.

(10) Kobayashi, H.; Ogawa, M.; Alford, R.; Choyke, P. L.; Urano, Y. New strategies for fluorescent probe design in medical diagnostic imaging. Chem. Rev. 2010, 110, 2610-2640.

(11) Lakowicz, J. R. Principles of fluorescence spectroscopy. 3rd ed. Springer. 2006.

(12) Leen, V.; Gonzalvo, V. Z.; De Borggraeve, W. M.; Boens, N.; Dehaen, W. Direct functionalization of BODIPY dyes by oxidative nucleophilic hydrogen substitution at the 3-or 3,5-positions. Chem. Commun. 2010, 46, 4908-4910.

(13) Filarowski, A.; Lopatkova, M.; Lipkowski, P.; Leen, V.; Van der Auweraer, M.; Dehaen, W. Solvatochromism of BODIPY-Schiff Dye. J. Phys. Chem. B. 2015, 119, 2576-2584. 
(14) Xiao, S.; Zou, Y.; Wu, J.; Zhou, Y.; Yi, T.; Li, F.; Huang, C. Hydrogen bonding assisted switchable fluorescence in self-assembled complexes containing diarylethene: controllable fluorescent emission in the solid state. J. Mater. Chem. 2007, 17, 2483-2489.

(15) Pan, Z.-H.; Zhou, J.-W.; Luo, G.-G. Experimental and theoretical study of enol-keto prototropic tautomerism and photophysics of azomethine-BODIPY dyads. Phys. Chem. Chem. Phys. 2014, 16, 16290-16301.

(16) Deniz, E.; Isbasar, G. C.; Bozdemir, O. A.; Yildirim, 1. T.; Siemiarczuk, A.; Akkaya, E. U. Bidirectional switching of near IR emitting boradiazaindacene fluorophores. Org. Lett. 2008, 10, 3401-3403.

(17) Coskun, A.; Deniz, E.; A.; Akkaya, E. U. Effective PET and ICT switching of boradiazaindacene emission: a unimolecular, emission-mode, molecular half-subtractor with reconfigurable logic gates. Org. Lett. 2005, 7, 5187-5189.

(18) Lakshmi, V.; Ravikanth, M. Boron-dipyrromethene based multi-anionic sensor and a specific cationic sensor for Fe3+. J. Mater. Chem. C 2014, 28, 5576-5586.

(19) Emsley, J.; Freeman, N. J. $\beta$-diketone interactions: Part 5. Solvent effects on the keto $\leftrightharpoons$ enol equilibrium. J. Mol. Struct. 1987, 161, 193-204.

(20) Grushow, A.; Zielinski, T. J. Hydrogen bonding using NMR: A new look at the 2,4pentanedione keto-enol tautomer experiment. J. Chem. Educ. 2002, 79, 707-714.

(21) Hohenberg, P.; Kohn, W. Inhomogeneous Electron gas. Phys. Rev. B 1964, 136, B864B871.

(22) Bauernschmitt, R.; Ahlrichs, R. Treatment of Electronic Excitations within the Adiabatic Approximation of Time Dependent Density Functional Theory. Chem. Phys. Lett. 1996, 256, $454-464$. 
(23) Zhao, Y.; Truhlar, D. G. The M06 suite of density functionals for main group thermochemistry, thermochemical kinetics, noncovalent interactions, excited states, and transition elements: two new functionals and systematic testing of four M06-class functionals and 12 other functionals. Theor. Chem. Acc. 2008, 120, 215-241.

(24) Perdew, J. P.; Burke, K.; Ernzerhof, M. Generalized Gradient Approximation Made Simple. Phys. Rev. Lett., 1996, 77, 3865-3868

(25) Yanai, T.; Tew, D.; Handy, N. A new hybrid exchange-correlation functional using the Coulomb-Attenuating Method (CAM-B3LYP). Chem. Phys. Lett. 2004, 393, 51-57.

(26) (a) Becke, A. D. Density-Functional Thermochemistry. III. The Role of Exact Exchange. $J$. Chem. Phys. 1993, 98, 5648-5652. (b) Lee, C.; Yang, W.; Parr, R. G. Development of the ColleSalvetti Correlation-Energy Formula into a Functional of the Electron Density. Phys. Rev. B. 1988, 37, 785-789.

(27) Cho, D. W.; Fujitsuka, M.; Ryu, J. H.; Lee, M. H.; Kim, H. K.; Majima, T.; Im, C. S2 Emission from Chemically Modified BODIPYs. Chem. Commun. 2012, 48, 3424-3426.

(28) Wang, Q.; Lu, H.; Gai, L.; Chen, W.; Lai, G.; Li, Zh. Emission of Boron Dipyrromethene Dyes through Energy Transfer to their S2 State from Polysilane S1 State. Dyes and Pigments 2012, 94, 183-186.

(29) De Simone, B. C.; Mazzone, G.; Pirillo, J.; Russo, N.; Sicilia, E. Halogen Atom Effect on the Photophysical Properties of Substituted aza-BODIPY Derivatives. Phys. Chem. Chem. Phys. 2017, 19, 2530-2536.

(30) Waluk, J. Hydrogen-Bonding-Induced Phenomena in Bifunctional Heteroazaaromatics. Acc. Chem. Res. 2003, 36, 832-838. 
(31) Waluk, J. Conformational Aspects of Intra- and Intermolecular Exited State Proton Transfer. In Conformational analysis of molecules in excited state; Waluk, J., Ed.; Wiley-VCH, 2000, pp 57-111. 